\title{
Origin of natural intelligence and conscience in humans and animals
}

\section{Opinion}

On the base of laws of physics, the natural evolution is a sort of intelligent machine that continuously solves problems and gives solutions. The physical variable at the basis of the biomimetic solutions is the improvement of the synergies in multi-element system that leads to more efficient conditions. The final system is able to produce the same output with minor expenses, or a more complex one with the same cost. This works for living structures and for related social dynamics.

Beyond the second law of thermodynamics, there is a new physical law that overcomes chaos and generates life and creativity. On this base it is possible to build a scientific and objective code for the evaluation of the intrinsic value of intelligence and conscience.

The principle of maximum energy dissipation allows the matter to organize itself far from equilibrium. The energetic fluxes determine the living matter architecture where the complexity of shape is linked to the high specialization of functions. The driver of the process is the energy and the matter is just the substrate. On this base, the living structures realize a sort of wetware (living tissues are biphasic material made of a solid network permeated by a liquid phase where fluxes of ions and molecules are possible) where energy and organization/information are handled.

The energetic outputs can be divided in two categories:

i. Macroscopic movements (e.g., muscle contraction).

ii. Microscopic ones (plastic rearrangement, electrochemical dynamics, transportation, rearrangement, storage of matter and information handling) both inside the cell for its functioning and outside in the extracellular matrix.

The biological intelligence is a product of these basic processes where the wetware (matter substrate) allows the production of the energy-matter fluxes and the energy-matter fluxes modify the mattersubstrate.

Many and perhaps the most important functions in a living system are generated by the inverse process of material modeling due to the energetic fluxes. The biphasic structuring is the step that, thanks to spatial separation, has allowed the generation of specialized functions in different places of the living structure. In this way it is possible to have every single part devoted to a particular purpose whose output is useful for sustaining the processes in the other places, all in circle. This has led to the development of the establishment of a network of synergies: the real step that generates the transition from organized structures to living systems.

This can be well understood by the following thought experiment: if we take away, from their position, all cells of a living system and then we put them back reversibly in such a way to re-obtain the starting state, we will observe that, when all the cells were far away each other, all living functions were suspended. Between those separated parts, both the energy-matter fluxes and the associated complexes functions
Volume 7 Issue 5 - 2018

\author{
Piero Chiarelli \\ Biomedical Engineering, University of Pisa, Italy
}

Correspondence: Piero Chiarelli, National Council of Research of Italy, Faculty of Biomedical Engineering University of Pisa, Italy, Email pchiare@ifc.cnr.it

Received: July 24, 2017 | Published: September 20, 2018

cannot be established. The life functions would be resumed only after the re-composition of the system. Hence, the only possible answer the question "of what about is the life composed by?" is: The life realizes itself in the interrelations between the parts by the synergic functions developed among organized components.

The wetware of the material substrate (bi-phasic means), where information is handled, allows the establishment of the energymatter fluxes that modify and organize the matter-substrate at each "informational state", it corresponds a material state that is a (rewritable) imprint of it. Moreover, since the numerous informational functions cannot be performed contemporaneously, all the processes are synchronized with respect to a biological clock. The first consequence is that the perception of the reality is not continuous and the time is discretized. The main demonstration of this fact is the realization of the cinemascope that is based upon this cerebral mechanism. The intellective output is then the "reaction" produced by the changes of the brain state (due to its plasticity generated by the energy-matter fluxes) to an external input, leading to evaluation of facts and to the plan of action. At the base of the "decision making" process there is the so called "conscience" of what "I am" and what "I can do" that the system has acquired with the experience. This process is based upon the physical predictability of the reality in the sense that if I observe a behavior following a certain input, I know that to a such premise it will follow a defined fact: The existence of the physical laws and the repeatability of the experiences are at the base of the formation of the conscience. In this sense I know what I am and what are my potentiality (so that I can understand where a chain of events can bring me). Moreover, since the imprints, where all the experienced states are written in our brain, are compared each other sequentially, the dynamics of our evolution is captured and a dynamical conscience is obtained by the perception of the differences.

The deepness of conscience, intelligence and free will are greatly influenced by the power of calculus of the mind that is only a part of the intellective process. Higher is the calculus power of the mind, longer is the time-horizon to which we can extend our previsions and hence to free decide what to do at the present: thence the old question does the free will exist or not, is basically a bad posed question, the correct answer is that the free willing exists but it is limited in time. The man is not omnipotent, but it has a finite power to act. 
Animals, at high level of evolutionary scale, are still able to understand the cause-effect process, the difference is their power of calculus that is smaller than in humans so that the time-horizon for the evaluations of the consequences of their acts is shorter and their conscience more limited but not null. The power of calculation has an explicit sign in the complexity of expression (about which the speaking is the maximum expression) that leads to the ability of exchange higher and higher amount of information among individuals.
The intelligence, as the ability to adapt to the environment and to act in agreement with it in order to obtain the maximum of synergies, is something wider than "power of calculus" and has its base in the emotional planning that the living systems adopt to respond to external stimuli. Sometime, the animals can be smarter than Humans in doing this job. 